\title{
Establishment and Clinical Features in Spontaneously Diabetic Torii Rat
}

\author{
Masami Shinohara*
}

\author{
CLEA Japan, Inc., Planning and Development Section, 1-2-7, Higashiyama, Meguro-ku, Tokyo 153-8533, Japan
}

\begin{abstract}
The Spontaneously Diabetic Torii (SDT) rat is a spontaneous animal model of non-obese Type 2 diabetes (T2D) resembling those of humans, established in 1997. I investigated the clinical features of the SDT rats. The time of onset of glucosuria was different between male and female SDT rats; glucosuria appeared at approximately 20 weeks of age in male rats and at approximately 45 weeks of age in female rats. A cumulative incidence of diabetes of $100 \%$ was noted by 40 weeks of age in male rats, while it was only $33.3 \%$ even by 65 weeks of age in female rats. The survival rate up to 65 weeks of age was $92.9 \%$ in male rats and $97.4 \%$ in female rats. The male SDT rats were (1) hyperglycemia and hypoinsulinemia (from 25 weeks of age); (2) a significant increase in urea nitrogen levels, urinary protein excretion and HbA1c levels (from 35 weeks of age); (3) long-term survival without insulin treatment after onset of diabetes; additionally, no obesity was noted in any of the male and female rats. These results indicated that the SDT rat strain described here would serve as useful animal model for studies of non-obese T2D.
\end{abstract}

Keywords: Type 2 diabetes, non-obese, Spontaneously Diabetic Torii strain, animal model, long-term survival.

\section{INTRODUCTION}

There are growing numbers of diabetic patients in all countries of the world, including developing countries, and these patients present worldwide care coverage issues. International Diabetes Federation has estimated that world diabetes population will reach 366 million in the year 2030 $[1,2]$. In addition, there has been an alarming increase in the incidence of type 2 diabetes (T2D) in children and adolescent [3, 4]. Various studies on the complicated interactions between genetic and environmental factors for the development of diabetes mellitus (DM) are currently underway. However, there are natural limits to the information that can be directly obtained from humans, partly because it is extremely difficult to demonstrate the relationship between these factors in humans and partly because collection of data from diabetic patients is subject to significant ethical restrictions. It is believed that the use of experimental animal models is essential for solving this problem. It is proposed that data from basic studies conducted in animal models of DM will be useful for elucidating the mechanism of development of diabetes in humans, studying the causes of diabetic complications, and developing medications for diabetes treatment. T2D models such as $o b / o b$ mice [5], KK mice [6], $d b / d b$ mice [7], TSOD mice [8], Goto-Kakizaki (GK) rats [9], Wistar fatty rats [10] and Otsuka-Long-Evans-Tokushima-Fatty (OLETF) rats [11] are also well known. Thus, results with implications for clinical diabetes have been obtained to date from many diabetic animal models. The development of models for T2D similar to those in human diabetes calls for urgent attention, partly because it is extremely important to develop animal models with a variety of pathologic conditions

*Address correspondence to this author at the CLEA Japan, Inc., Planning and Development Section, 1-2-7, Higashiyama, Meguro-ku, Tokyo 1538533, Japan; Tel: +81-35704-7272; Fax: +81-33791-2859;

E-mails: shinohara-m@clea-japan.com, aaron@asahi-ns.email.ne.jp corresponding to human diabetes and partly because in recent years, T2D has increased rapidly and globally along with economic development and social modernization. Because of this situation, we have newly established a nonobese T2D model rat with diabetic complications resembling those of humans, this model develops diabetic retinopathy [12-19], diabetic peripheral neuropathy [20,21] and diabetic nephropathy $[22,23]$.

\section{ESTABLISHMENT OF THE SDT STRAIN}

In 1988, twelve-month-old male rats that exhibited polydipsia, polyphagia, polyuria, and glucosuria were discovered among Sprague-Dawley (SD) rats from Charles River Japan by Shinohara at the Research Laboratories of Torii Pharmaceutical Co., Ltd., Japan. Male rats were mated with normal female rats of the same strain to maintain the disease symptoms, and in 1991 several rats were found to exhibit positive urinary glucose earlier than previous strains (at four to five months of age). An inbred strain of a nonobese T2D model was established by repeated sister-brother mating, and the animals obtained were named "Spontaneously Diabetic Torii" (SDT) rats in 1997 [12, 13] (Fig. 1). Male rats presented DM from the $F_{1}$ to the $F_{20}$ generation. On the other hand, diabetic female rats were observed from the $F_{7}$ generation. Fourteen $F_{20}$ rats were obtained, of which there were 5 diabetic males and 8 diabetic females from the nucleus lines of the SDT strain [13] (Fig. 2). All rats were kept in our specific pathogen-free (SPF) facilities, which were also free from the following microorganisms: Sendai virus, Sialodacryoadenitis virus, the pneumonia virus of mice, mouse encephalomyelitis virus, Kilham rat virus, $\mathrm{H}-1$ virus, the minute virus of mice Hantavirus, mouse adenovirus, Mycoplasma pulmonis, Bacillus piriformis, CAR bacillus, Bordetella bronchiseptica, Corynebacterium kutscheri, Pasteurella pneumotropica, Pseudomonas aeruginosa, Salmonella spp, Salmonella typhimurium, Streptococcus pneumoniae, Dermatophytes, Giardia muris, Spironucleus muris and Syphacia spp. Since 
these rats are inherently gentle in nature throughout their lifetime, they were easy to handle for breeding and experiments $[12,13]$.

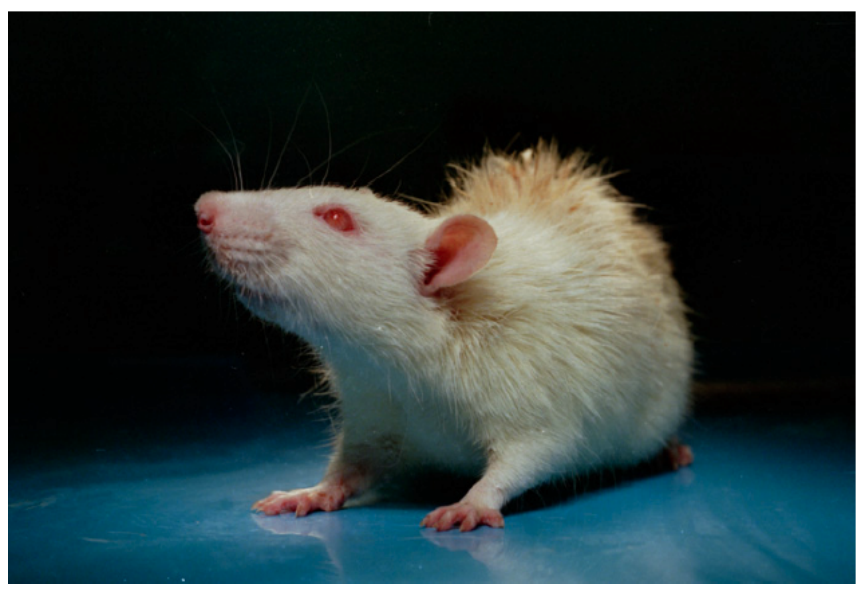

Fig. (1). Appearance of a male SDT rat aged 35 weeks.

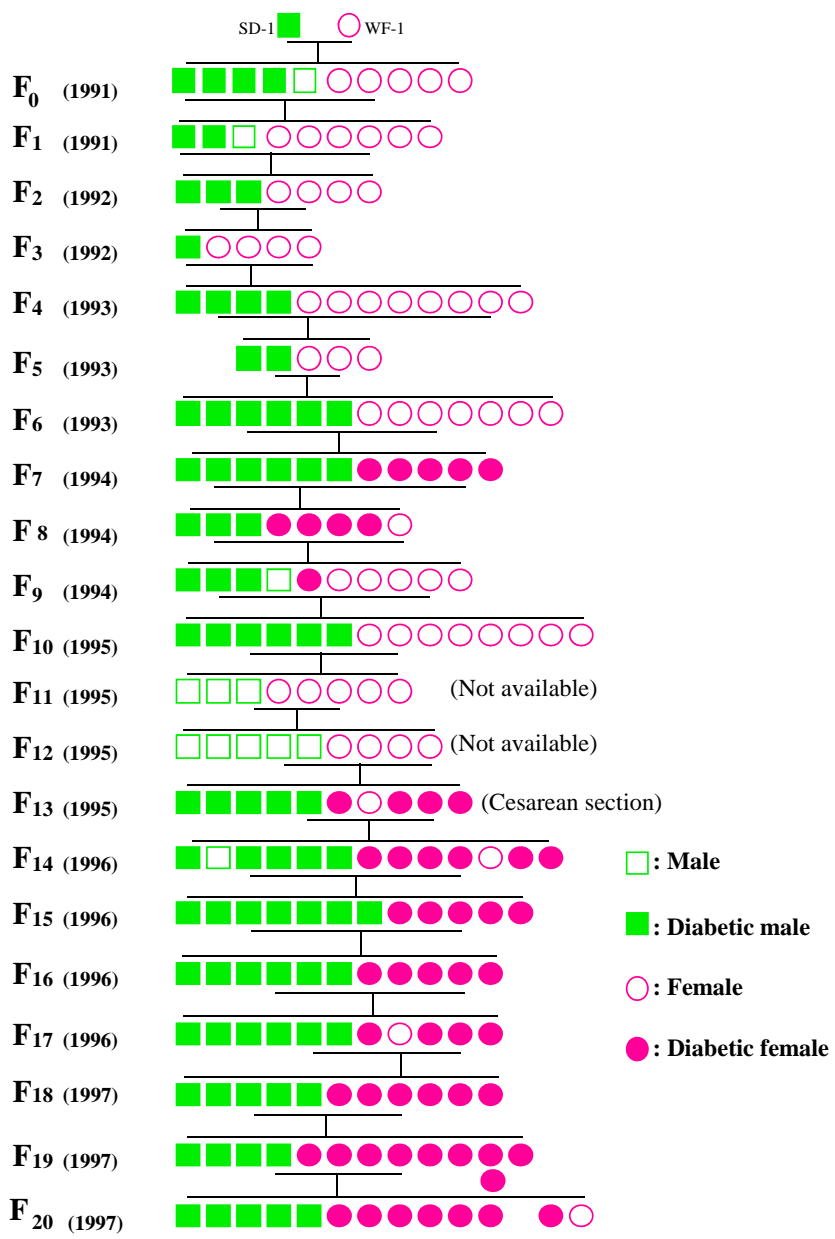

Fig. (2). Pedigree maps of nucleus line of SDT rats.

\section{CLINICAL FEATURES OF THE SDT RAT}

The average body weight of male SDT rats was slightly lower than that of SD rats until 18 weeks of age and then gradually decreased as the incidence of diabetes increased. A significant decrease in body weight was observed beginning from 20 weeks of age. On the other hand, from 6 to 40 weeks of age the average body weight of female SDT rats increased gradually throughout the experimental period, and the changes in body weight in female SDT rats were similar to those found in female SD rats. The non-fasting plasma glucose levels in male SDT rats reached $701 \pm 103 \mathrm{mg} / \mathrm{dl}$ by 25 weeks of age and the values gradually increased with advancing ages, whereas those in SD rats were maintained at a steady level. Plasma glucose levels in female SDT rats were slightly but significantly higher than those in female SD rats after 10 weeks of age. The plasma insulin levels of male SDT rats were significantly lower than those in 25week-old SD rats. Furthermore, the plasma urea nitrogen levels, urinary protein excretion and HbAlc levels in male SDT rats were $1.4,3.9$ and 3.5 times higher respectively than those in SD rats at 35 weeks of age. Glucosuria appeared at 20 weeks of age in male SDT rats (15/42), and the time of onset of glucosuria was coincident with that of plasma glucose elevation. The cumulative incidence of diabetes increased sharply, to $52.4 \%$ in 25 -week-old-rats (22/42) and $100 \%$ in 40 -week-old rats $(42 / 42)$. Glucosuria had not yet appeared in female SDT rats even by 40 weeks of age. It was first observed at 45 weeks of age, and thereafter, the incidence of diabetes gradually increased. However, the maximal incidence of $33.3 \%$, noted in female rats at 65 weeks of age, was markedly lower than that noted in male SDT rats. These findings suggest that sex hormones are important in the development of DM in this model rat. The symptoms polyuria, polydipsia and polyphagia were also observed in both sexes of SDT rats after onset of diabetes. However, no ketonuria was noted up to 65 weeks of age in male or female SDT rats. The survival rates of male and female SDT rats up to 65 weeks of age were $92.9 \%(39 / 42)$ and $97.4 \%$ (38/39), respectively. The first deaths among male and female SDT rats were observed at 40 weeks of age and 45 weeks of age, respectively $[12,13]$.

\section{CONCLUSION}

In conclusion, the important findings from this study that especially in male SDT rats show $100 \%$ incidence of diabetes in 40-week-old; moreover, the diabetic rats show typical symptoms of DM. In addition, the survival rates of the male and female SDT rats were very long-term. Although there are many T2D model animals, newly established SDT rats show symptoms similar to those in human diabetes and very useful for the investigation of nonobese T2D.

\section{NOTES ON REARING AND HANDRING SDT RATS}

\section{Diet and Drinking Water}

In SDT rats, glucosuria appears on ingestion of standard feed (CE-2, CLEA Japan, Tokyo, Japan) for mice and rats. Their feed intake increases (to about 1.5 to 2 times that of SD strain rats) and their water intake also increases significantly (200 to $400 \mathrm{ml} /$ day and more) with the onset of diabetes. It is, therefore, important to always supply sufficient amounts of feed and drinking water.

\section{Frequent Changing of Bedding and Cleaning of Cages are Recommended}

After the onset of diabetes, the SDT rats produce large volumes of urine and their bedding easily becomes dirty. Therefore, they can easily develop urinary tract infections. In order to avoid such infections, each rat should be reared in 
an individual plastic cage with a large amount of bedding. It is also recommended to change bedding frequently in case of bracket cage rearing.

\section{DISTRIBUTOR INFORMATION FOR SDT RATS}

CLEA Japan, Inc.

1-2-7, Higashiyama, Meguro-ku, Tokyo 153-8533, Japan

Tel: 81-35704-7272

Fax: 81-33791-2859

E-mail: export@clea-japan.com

URL: http://www.clea-japan.com

\section{ACKNOWLEDGEMENTS}

The author is grateful to Dr. Taku Masuyama, Dr. Akihro Kakehashi, Dr. Toshiyuki Shoda, Dr. Kajuro Komeda and Dr. Yasunori Kanazawa for their contributions to this work.

\section{REFERENCES}

[1] Zimmet P, Alberti KG, Shaw J. Global and societal implications of the diabetes epidemic. Nature 2001; 414: 782-7.

[2] Wild S, Roglic G, Green A, Sicree R, King H. Global prevalence of diabetes: estimates for the year 2000 and projections for 2030 . Diabetes Care 2004; 27: 1047-53.

[3] Shaw J. Epidemiology of childhood type 2 diabetes and obesity. Pediatr Diabetes 2007; 8(Suppl 9): 7-15.

[4] Nadeau K, Dabelea D. Epidemiology of type 2 diabetes in children and adolescents. Endocr Res 2008; 33: 35-8.

[5] Colman DL, Hummel KP. The influence of genetic background on the expression of the obese (ob) gene in the mouse. Diabetologia 1973; 9: 287-93.

[6] Dulin WE, Wyse BM. Diabetes in the KK mouse. Diabetologia 1970; 6: 317-23.

[7] Colman DL, Hummel KP. Hyperinsulinemia in pre-weaning diabetes (db) mice. Diabetologia 1974; 10: 607-10.

[8] Suzuki W, Iizuka S, Tabuchi M, et al. A new mouse model of spontaneous Diabetes derived from ddY stain. Exp Anim 1999; 48: $181-9$.

[9] Goto Y, Suzuki K, Ono T, Sasaki M, Toyota T. Development of diabetes in the non-obese NIDDM rat (GK rat). Adv Exp Med Biol 1988; 246: 29-31.
[10] Ikeda H, Shino A, Matsuo T, Iwatuka H, Suzuoki Z. A new genetically obese-hyperglycemic rat (Wistar Fatty). Diabetes 1981; 30: $1045-50$.

[11] Kawani K, Hirashima T, Mori S, Saitoh Y, Kurosumi M, Natori T. Spontaneous long-term hyperglycemic rat with diabetic complications. Otsuka Long-Evans Tokushima Fatty (OLETF) Strain. Diabetes 1992; 41: 1422-8.

[12] Shinohara M, Masuyama T, Shoda T, et al. A new spontaneously diabetic non-obese Torii rat strain with severe ocular complications. Int J Exp Diabetes Res 2000; 1: 89-100.

[13] Shinohara M, Masuyama T, Kakehashi A. The spontaneously diabetic Torii (SDT) rat with retinopathy lesions resembling those of humans. In Shafrir E, Eds. Animal Models of diabetes: frontiers in research. 2nd ed. Boca Ration, FL, USA: CRC Press 2007; pp. 311-21.

[14] Yamada H, Yamada E, Higuchi A, Matsumura M. Retinal neovascularisation without ischaemia in the Spontaneously Diabetic Torii rat. Diabetologia 2005; 48: 1663-8.

[15] Kakehashi A, Saito Y, Mori K, et al. Characteristics of diabetic retinopathy in SDT rats. Diabetes Metab Res Rev 2006; 22: 45561.

[16] Sasase T, Ohta T, Ogawa N, et al. Preventive effects of glycaemic control on ocular complications of the Spontaneously Diabetic Torii rat. Diabetes Obes Metab 2006; 8: 501-7.

[17] Shoda T, Shinohara M, Takahashi T, Miyajima K, Kakehashi A, Miyakawa Y. Histopathological features of diabetic ocular complications in the Spontaneously Diabetic Torii (SDT) rat. J Toxicol Pathol 2007; 20: 179-83.

[18] Okuno T, Oku H, Sugiyama T, Ikeda T. Electroretinographic study of Spontaneously Diabetic Torii rat. Doc Ophthalmol 2008; 117: 191-96.

[19] Fukuda M, Nakanishi Y, Fuse M, et al. Altered expression of aquaporins 1 and 4 coincides with neurodegenerative events in retinas of spontaneously diabetic Torii rats. Exp Eye Res 2010; 90: $17-25$.

[20] Sasase T, Ohta T, Ogawa N, et al. Diabetic complications of the Spontaneously Diabetic Torii rat. Diabetes 2005; 54(Suppl 1): A221.

[21] Sasase T, Morinaga H, Abe T, et al. Protein kinase C beta inhibitor prevents diabetic peripheral neuropathy, but not histopathological abnormalities of retina in the Spontaneously Diabetic Torii rat. Diabetes Obes Metab 2009; 11: 1084-87.

[22] Ohta T, Matsui K, Miyajima K, et al. Effect of insulin therapy on renal changes in spontaneously diabetic Torii rats. Exp Anim 2007; 56: $355-62$.

[23] Fujii H, Kondo K, Nakai K, et al. Oxidative and nitrosative stress and progression of diabetic nephropathy in type 2 diabetes. Am J Nephrol 2010; 31: 342-52. 